\title{
Evaluation of the effects of various gas chromatographic parameters on haloacetic acids disinfection by-products analysis
}

\author{
Yee-Chung Ma*, Chi-Yung Chiang \\ Institute of Environmental Health, College of Public Health, National Taiwan University, No. 1 Jen-Ai Road, Section 1, Taipei, Taiwan
}

Received 5 January 2005; received in revised form 22 February 2005; accepted 23 March 2005

\begin{abstract}
Thermal decomposition of the analytes during the analysis is a problem for haloacetic acids (HAAs). We evaluated the effect of GC injection port temperature and the amount of trace water in the sample on the HAAs' analysis. For three brominated HAAs, the variation in intensity due to the change of injection port temperature was significant. The largest variation observed was tribromoacetic acid methyl ester (from 3.2 to 1 for injection port temperature changing from $170^{\circ} \mathrm{C}$ to $250^{\circ} \mathrm{C}$ ). Tribromoacetic acid methyl ester partially decomposed to dibromoacetic acid and to tribromomethane in a competitive way. At a low injection port temperature, tribromomethane formation was preferred, but at a high injection port temperature, the debrominated methyl ester formation dominated. Water contained in the sample may accelerate the hydrolysis process of the esters in the injection port, and this effect was also the greatest for the brominated trihaloacetic acids. Direct injection of underivatized HAAs into the GC/MS indicated that brominated HAAs can be nearly quantitatively thermal decomposed to the corresponding halomethanes.
\end{abstract}

(C) 2005 Elsevier B.V. All rights reserved.

Keywords: Haloacetic acids; Derivatization; GC/MS parameters

\section{Introduction}

Thermal decomposition of an organic compound during sample preparation and instrumental analysis has been observed frequently. Examples include the loss of water from relatively involatile alcohols, the loss of carbon dioxide from dicarboxylic acids, and the decomposition of benzophenone oximes to corresponding imines and benzophenones, etc. [1-4]. Compounds that are subject to thermal decomposition may not be accurately or reproducibly quantitated by different laboratories due to the differences in laboratory skills, practices, or instrument parameters. This paper is focused on the effects of thermal decomposition on the gas chromatography/mass spectrometry (GC/MS) analysis of the haloacetic acids as a by-product of water disinfection.

\footnotetext{
* Corresponding author. Tel.: +886 2 23123456x8473; fax: +886223418577 .

E-mail address: ycma@ha.mc.ntu.edu.tw (Y.-C. Ma).
}

Haloacetic acids are comprised of a total of nine species (HAA9), of which only five (HAA5) have their maximum contaminant levels (MCL) set in the 1998 USEPA D/DBP Rule $(60 \mathrm{ug} / \mathrm{L})$ [5]. These include monochloroacetic acid (mCAA), dichloroacetic acid (dCAA), trichloroacetic acid (tCAA), monobromoacetic acid (mBAA), and dibromoacetic acid (dBAA). As a result, the literatures reporting on the analysis of haloacetic acids are mostly were focused on these five species. The remaining four species, bromochloroacetic acid (BCAA), bromodichloroacetic acid (dCBAA), chlorodibromoacetic acid (dBCAA), and tribromoacetic acid (tBAA) are often ignored in these analyses. This is partly due to their relatively low formation probability, and therefore, the less chance of being encountered in a common situation and partly due to problems of the analytical methods.

The most commonly used methods for the analysis of HAAs were using liquid-liquid extraction, chemical derivatization, followed by GC/electron capture detector (ECD) or GC/MS analysis, such as the US Environmental Protection Agency (EPA) methods 552.1, 552.2, and standard method 
6251B [6-8]. EPA method 552.2 is used to analyze all nine haloacetic acid species, and includes three major steps: (1) liquid-liquid extraction, (2) chemical derivatization, and (3) analysis with GC/ECD or GC/MS. Because trihaloacetic acids are thermally speaking, relatively unstable, even the EPA methods 552.1 or 552.2 point out that high temperature derivatization and extraction will lead to the formation of trihalomethanes from the thermal decomposition of trihaloacetic acid methyl ester, and result in an inaccurate analysis. Since thermally unstable compounds can also decompose in the high temperature GC injection port, especially when in the splitless injection mode, the GC parameters used during GC analysis are another factor affecting the accuracy of the final results. This study investigated and evaluated several GC injection port operation parameters that have the potential to cause an inaccurate analysis. The parameters investigated include injection port temperature variation, and the effect of residual water in the esterfied sample. We also include an interesting result which we observed during the analysis, that of direct injection of underivatized haloacetic acids.

\section{Experimental}

\subsection{Reagents and standards}

Haloacetic acids and haloacetic acid methyl esters (in methyl tertiary-butyl ether, MTBE; all purity, >98.0\%) were obtained from Chem Service (West Chester, PA, USA). Internal standard 1,2,3-trichloropropane (tCP; purity, >98\%) was obtained from Actu Standard (New Heaven, CT, USA). Halomethanes were also obtained from Chem Service (all purity, >98\%). HPLC grade MTBE was obtained from Fluka (Buchs, Switzerland). Double deionized reagent water was obtained from in house Millipore Milli-Q water purification system (Millipore, Beldford, MA, USA).

\subsection{Sample preparation}

Standard samples ready for GC injection, either as pure forms or as mixtures, were prepared by mixing appropriate amounts of original standards and diluted to $1000 \mathrm{ug} / \mathrm{mL}$ stock solutions. Stock solutions were then serial diluted to $20 \mathrm{ug} / \mathrm{mL}$ stock solutions. One $\mathrm{ng} / \mathrm{uL}$ internal standard tCP was used in the experiment, and MTBE was used for all the dilution.

\subsection{GC/MS analysis}

The experiments were performed with an Agilent model 6890 gas chromatograph, equipped with a 5973 mass-selective detector (MSD). Sample was injected instantaneously by the autosampler. Compounds were separated using a DB- 1 capillary column $(60 \mathrm{~m} \times 0.25 \mathrm{~mm}$ i.d., film thickness $0.25 \mathrm{um}$ ) (Wilmington, DE, USA). Splitless mode injection was used in the experiment with a 1 min purge on time. Helium (99.999\% purity) was used as the carrier gas at $10 \mathrm{psi}$ head pressure. The GC oven temperature was programmed as follows: initial temperature of $40^{\circ} \mathrm{C}$ (unless in the initial oven temperature variation experiment), hold for $4 \mathrm{~min}$, increase to $70^{\circ} \mathrm{C}$ at $3{ }^{\circ} \mathrm{C} / \mathrm{min}$, hold for $3 \mathrm{~min}$, then increase to $110^{\circ} \mathrm{C}$ at $5^{\circ} \mathrm{C} / \mathrm{min}$, then a final increase to $220^{\circ} \mathrm{C}$ at $30^{\circ} \mathrm{C} / \mathrm{min}$ to bake out any residuals. The GC/MS transfer line is maintained at $280^{\circ} \mathrm{C}$. Full scan (from $35 \mathrm{amu}$ to $350 \mathrm{amu}$ ) mode is used in the study.

\subsubsection{GC injection port temperature variation}

One microliter of $10 \mathrm{ng} / \mathrm{uL}$ individual haloacetic acid methyl ester standard solutions were injected into GC/MS at seven different injection port temperatures $\left(170^{\circ} \mathrm{C}\right.$, $180^{\circ} \mathrm{C}, 190^{\circ} \mathrm{C}, 200^{\circ} \mathrm{C}, 210^{\circ} \mathrm{C}, 230^{\circ} \mathrm{C}$, and $250^{\circ} \mathrm{C}$ ). For the detection of methyl esters and halomethanes, a full scan (from $\mathrm{m} / z 35$ to $\mathrm{m} / \mathrm{z}, 350$ ) was used during the analysis, and the integrated peak areas were used to compare the injection port temperature's effect on individual species.

\subsubsection{Effect of trace water in the sample on the GC/MS analysis}

A 1:9 water and HAA9 mixture (100 ng/uL) was mixed, and $1 \mathrm{uL}$ of the mixture was injected into the GC/MS under splitless mode condition, and the result was compared with the same concentration HAA9 mixture standard without water.

\subsubsection{Direct analysis of haloacetic acids}

Haloacetic acids standard solutions were directly injected into the GC/MS under splitless mode condition, and the corresponding decarboxylated halomethanes were monitored.

\section{Results and discussion}

\subsection{Effect of injection port temperature on the haloacetic acid methyl esters analysis}

A mixture of nine ester standard was injected into the $\mathrm{GC}$ under different injection port temperatures $\left(170-250^{\circ} \mathrm{C}\right)$ using the splitless mode injection technique. Products were analyzed using a MS detector. The variations in peak areas of the esters are shown in Fig. 1. All nine species showed a decreased peak area at elevated injection port temperatures. However, the degree of decrease was significantly different for three trihaloacetic acid methyl esters containing bromine. The correction of the absolute peak area to relative peak area with internal standard corrected the effect of the injection port temperature for all species except for these three bromine containing esters. Analysis of the GC/MS traces showed that trihalomethanes were formed in the process, evidence of thermal decomposition.

Individual haloacetic acid methyl ester was then injected to confirm this observation. Surprisingly, for some of the haloacetic acid methyl esters, actually two thermal decomposition products had formed. For example, 

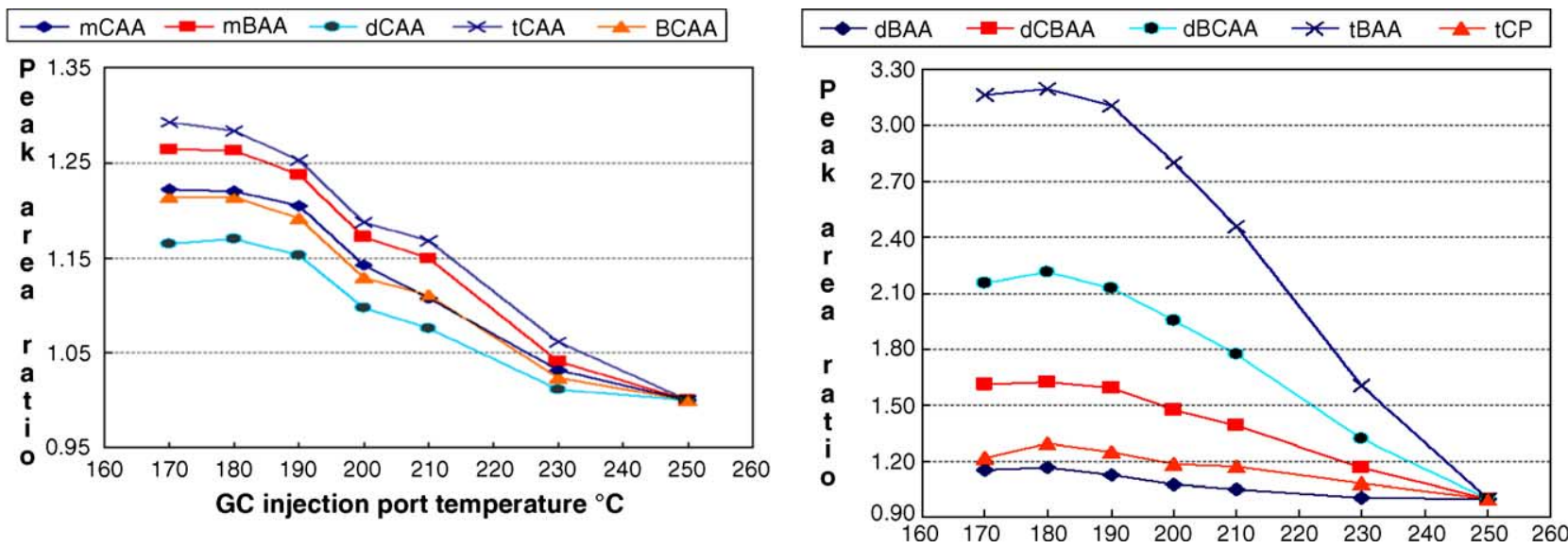

Fig. 1. Effect of GC injection port temperature on the HAA methyl ester signal. Ratio of peak area was used to demonstrate the large effect of GC injection port temperature on brominated trihaloacetic methyl esters. The peak area at $250{ }^{\circ} \mathrm{C}$ injection port temperature was used as the reference. Five nanograms $\mathrm{HAA}$ methyl esters were injected ( $1 \mathrm{ng}$ for internal standard tCP). For the abbrivation of each species, please see text, except note that these are methyl esters.
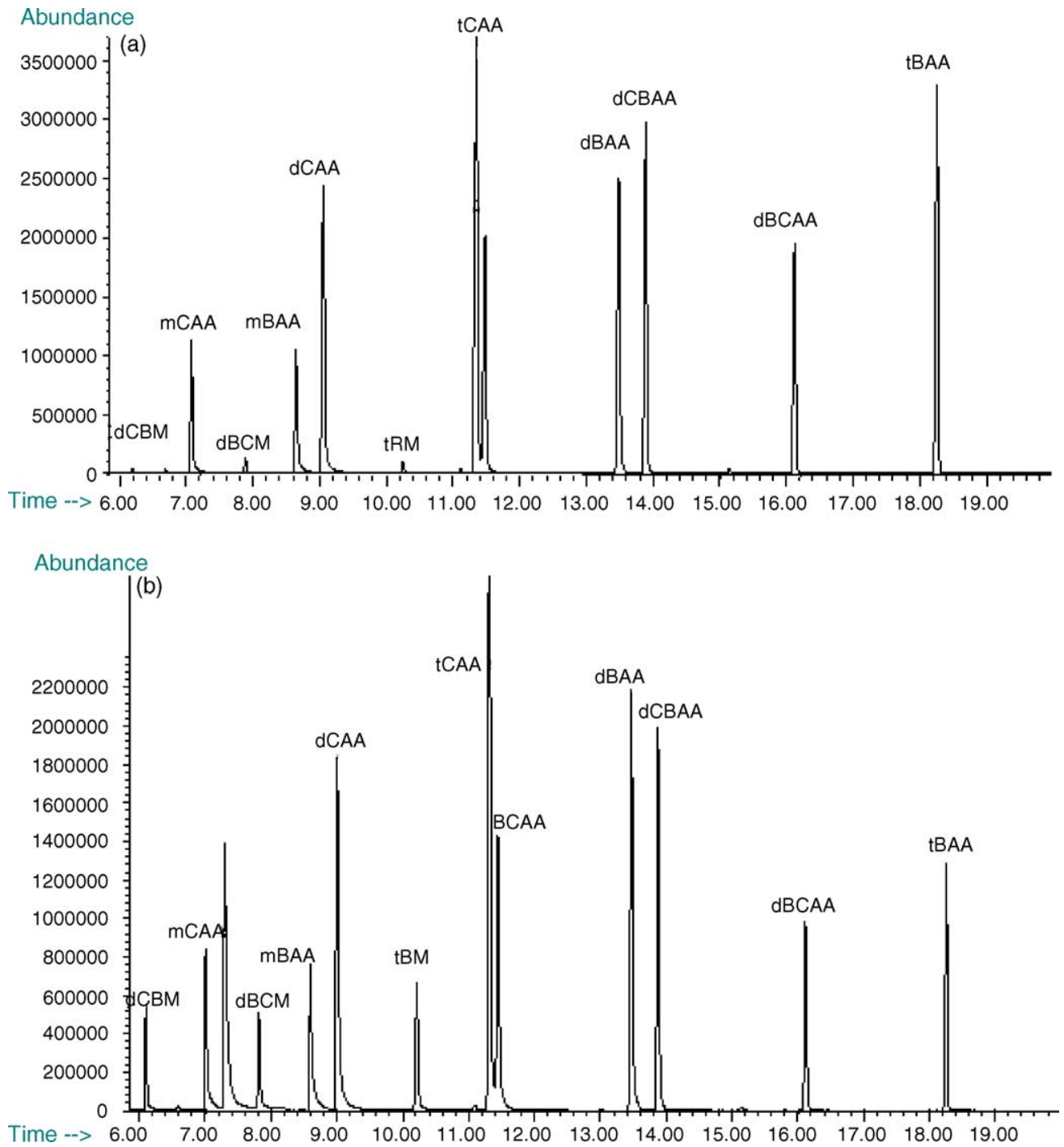

Fig. 2. Effect of water content in the sample on the GC analysis of HAA methyl ester: (a) without trace water and (b) with trace water. 
tribromoacetic acid methyl ester formed a debrominated methyl ester and a halomethane. At elevated injection port temperatures, a significant variation in peak areas was observed. The formation of the former product increased with the increase in injection port temperature, while the formation of the latter product decreased. Similar results were also observed for the other two brominated trihaloacetic acid methyl esters, monochlorodibromoacetic acid methyl ester and dichloromonobromoacetic acid methyl ester, forming monochloromonobromoacetic acid methyl ester and monochlorodibromomethane, and dichloroacetic acid methyl ester and dichloromonobromomethane, respectively.

The formation of debrominated species obviously has a mechanism that is different from that of the formation of trihalomethanes. These formations were ignored when the nine standards were injected as a mixture.

\subsection{Effect of trace water in the sample on the analysis}

Ester can be hydrolyzed to acid and alcohol when in contact with water. If there is any trace water in the sample, the hydrolysis may be accelerated at the hot injection inlet, once the sample is injected into the GC. Fig. 2 shows the comparison of the GC/MS chromatograms of two HAA methyl ester samples, with and without water content. Water content decreased the sensitivity of all the species, but the effect was greatest for the brominated trihaloacetic acid methyl esters. Since halomethanes were formed in the process, it is reasonable to assume that esters were first hydrolyzed to acids, and that the acids were then decarboxylated to the corresponding halomethanes. Whether the hydrolysis step occurred in the hot injection port or immediately after water was mixed with the standards, or which portion contributed the most was not determined.

\subsection{Direct injection and analysis of halo acetic acids}

Thermal decomposition of HAA methyl esters to the corresponding halomethanes in the injection port is of interest. These halomethanes can be formed by a one-step process or by a two-step processes first involving hydrolysis (to the corresponding acids) followed by decarboxylation. Regardless of the mechanism, if methyl esters can be thermally decomposed to halomethanes, we expect to observe similar and even stronger in underivatized acetic acids, especially for the brominated trihaloacetic acids. Direct injection of standard haloacetic acids into the GC/MS and monitoring of the corresponding halomethanes formed proved this assumption. The acetic acids could not be detected for all nine HAAs during the direct GC/MS analysis. However, for HAA3 and for trichloroacetic acid, the formation of the corresponding halomethanes was observed. The conversion of HAA3 to the halomethanes seemed nearly quantitatively, since direct injection of the same amounts of standard trihalomethanes gave fairly similar signal intensities in the GC/MS. We prepared seven different concentrations $(0.1-5 \mathrm{ug} / \mathrm{mL})$ of HAA3 and used their corresponding halomethane signals to construct individual's calibration curves, and obtained excellent straight lines $\left(R^{2}\right.$ all better than 0.995$)$. This feature probably can be incorporated into a future analysis scheme for the HAA9. For example, analyze HAA6 and HAA3 separately. The HAA6 can be detected as the methyl esters and the HAA3 can be detected as the corresponding thermal decomposition products (halomethanes). One of our proposed analysis schemes is to combine the analysis of trihalomethanes (THMs) with the analysis of HAA3: first, use purge-and-trap GC/MS to analyze and remove free trihalomethanes (THMs) and then extract HAA3 from the THMs-free sample for the direct injection analysis.

\section{Conclusions}

In the GC/MS analysis of the methyl ester derivatives of disinfection by-products haloacetic acids, use of a high GC injection port temperature increased the thermal decomposition phenomena, especially for the brominated trihaloacetic acid methyl esters. The thermal decomposition processes include debromination and decarboxylation which result in the formation of two products. Consequently, this results in the underestimation of the original methyl esters concentration, and the overestimation of the original concentration of the debrominated methyl esters products. Finally, the water content in the derivatized methyl ester sample may increase the rate of the methyl ester hydrolysis in the hot GC injection port thereby increasing the production of the corresponding halomethanes.

\section{References}

[1] G.E. von Unruh, W.D. Paar, Biom. Environ. Mass Spectrom. 13 (1986) 651.

[2] M.L. Deem, Org. Mass Spectrom. 15 (1980) 573.

[3] M. Bartoszek, D. Salzwedel, G. Stumm, H.J. Niclas, Org. Mass Spectrom. 22 (1987) 259.

[4] Y.C. Ma, B. Munson, Org. Mass Spectrom. 26 (1991) 82.

[5] United States Environmental Protection Agency, Federal Register 63 (241) (1998) 69390.

[6] J.W. Hodgeson, D. Becker, Methods for the Determination of Organic Compounds in Drinking Water, EPA method 552.1, Revision 1.0, EPA/600/R-92/129, Suppl. II, 1992.

[7] D.J. Munch, J.W. Munch, A.M. Pawlecki, Methods for the Determination of Organic Compounds in Drinking Water, EPA method 552.2, Revision 1.0, EPA/600/R-95/131, Suppl. III, 1995.

[8] A.D. Eaton, L.S. Clesceri, A.E. Greenburg (Eds.), Standard Methods for the Examination of Water and Wastewater, 19th ed., 1995. 